\title{
BMJ Open Neural biomarkers for assessing different types of imagery in pictorial health warning labels for cigarette packaging: a cross-sectional study
}

\author{
Roger D Newman-Norlund, ${ }^{1}$ James F Thrasher, ${ }^{2}$ Johann Fridriksson, ${ }^{2}$ \\ William Brixius, ${ }^{3}$ Brett Froeliger, ${ }^{4,5}$ David Hammond, ${ }^{6}$ Michael K Cummings ${ }^{5}$
}

To cite: Newman-

Norlund RD, Thrasher JF, Fridriksson J, et al. Neural biomarkers for assessing different types of imagery in pictorial health warning labels for cigarette packaging: a cross-sectional study. BMJ Open 2014;4:e006411. doi:10.1136/bmjopen-2014006411

- Prepublication history and additional material is available. To view please visit the journal (http://dx.doi.org/ 10.1136/bmjopen-2014006411).

Received 18 August 2014 Revised 24 November 2014 Accepted 25 November 2014

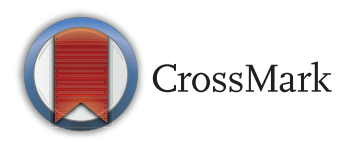

For numbered affiliations see end of article.

Correspondence to Dr Roger D NewmanNorlund;

rnorlund@mailbox.sc.edu

\section{ABSTRACT}

Objective: Countries around the world have increasingly adopted pictorial health warning labels (HWLs) for tobacco packages to warn consumers about smoking-related risks. Research on how pictorial HWLs work has primarily analysed self-reported responses to HWLs; studies at the neural level comparing the brain's response to different types of HWLs may provide an important complement to prior studies, especially if self-reported responses are systematically biased. In this study we characterise the brain's response to three types of pictorial HWLs for which prior self-report studies indicated different levels of efficacy.

Methods: Current smokers rated pictorial HWLs and then observed the same HWLs during functional MRI (fMRI) scanning. Fifty 18-50-year-old current adult smokers who were free from neurological disorders were recruited from the general population and participated in the study. Demographics, smokingrelated behaviours and self-reported ratings of pictorial HWL stimuli were obtained prior to scanning. Brain responses to HWLs were assessed using fMRI, focusing on a priori regions of interest.

Results: Pictorial HWL stimuli elicited activation in a broad network of brain areas associated with visual processing and emotion. Participants who rated the stimuli as more emotionally arousing also showed greater neural responses at these sites.

Conclusions: Self-reported ratings of pictorial HWLS are correlated with neural responses in brain areas associated with visual and emotional processing. Study results cross-validate self-reported ratings of pictorial HWLs and provide insights into how pictorial HWLS are processed.

\section{INTRODUCTION}

According to the WHO, smoking remains the leading cause of preventable death in the Western world. ${ }^{12}$ Smoking increases the risk of many non-communicable diseases in smokers as well as in those who breathe secondhand

\section{Strengths and limitations of this study}

- This is the first study to explore the relationship between self-reported ratings of pictorial health warning labels (HWLs) and neural responses to pictorial HWLs in a large sample $(\mathrm{N}=50)$ of current adult smokers.

- This paper demonstrates the amygdala is maximally activated by pictorial HWLS that depict human suffering, followed by images that depict graphic effects of smoking, followed by symbolic images of the negative consequences of smoking.

- This paper demonstrates that neural responses to pictorial HWLS attenuate with repeated exposure in most brain regions, but that this response is different in the amygdala.

- Further research is required in order to determine (1) exactly why pictorial HWLs depicting human suffering elicited such robust responses in the amygdala and (2) whether differential adaptation to symbolic stimuli is relevant to the creation of optimal HWLS.

smoke. ${ }^{3}$ To help prevent tobacco use and its consequences, the WHO Framework Convention on Tobacco Control (WHO FCTC) has recommended inclusion of prominent, pictorial health warning labels (HWLs) on tobacco packaging to communicate the adverse effects of smoking to consumers and to discourage smoking. ${ }^{2}$ Experimental and observational research indicate that HWLs with pictorial imagery are more effective than text-only HWLs in promoting smoking cessation and preventing the initiation of smoking behaviour. ${ }^{4-7}$ A key advantage of pictorial HWLs is their ability to elicit stronger emotional responses than text-only HWLs.

The increasing adoption of pictorial HWLs around the world has created a critical need for research designed to: (1) evaluate the relative effectiveness of different types of 
HWL content and (2) explain why some HWL content appears to be more effective than other content. Such research should guide the selection of HWL content, including the rotation of new HWL content over time. Some experimental research has found the self-reported effectiveness of pictorial HWLs is highest when it contains graphic images that depict the physical effects of smoking, followed by imagery of personal suffering (usually including a face), and finally by symbolic representations of smoking effects that use abstract imagery or symbols to represent risk. ${ }^{9-12}$ These findings are consistent with observational studies indicating that graphic depictions of smoking consequences work best. ${ }^{4}{ }^{13}$

The primary goal of the current experiment was to explicitly map neural responses to HWLs that contain three different subtypes of imagery that are frequently used in tobacco control communications, including HWLs on cigarette packaging: graphic representation of physical consequences of smoking; personal suffering from smoking-related consequences and symbolic representations of risk. Given the visual and emotional nature of pictorial HWLs, we formulated a set of a priori regions of interest (ROIs) that we expected to respond to participants' observations of HWLs, including the amygdala, insula and visual association cortex. Converging evidence from numerous neuroscientific investigations confirms a prominent role for the amygdala in emotional processing in a number of sensory modalities. ${ }^{14-18}$ The amygdala plays a particularly important role in the processing of visual stimuli related to threat and fear. ${ }^{19-21}$ We expected that amygdala responses would be driven by our stimuli to the extent that they elicited arousal, fear and perceived threat (eg, graphic HWL vs symbolic HWL). We also expected pictorial HWLs to elicit robust activity in the insula. This area has been linked to the experience of disgust, and strongly responds to pictures of mutilation and contamination. ${ }^{22-25}$ Finally, based on a prior investigations of the neural response to emotional pictures, we expected the visual association cortex to be robustly activated by the presentation of pictorial HWLs. ${ }^{26-28}$ We expected all three subtypes of HWLs to elicit a significant response (relative to rest) in this subset of a priori ROIs.

Our secondary goal was to examine the relationship between self-report data indicating that HWLs that use graphic imagery are more effective than HWLs depicting human suffering, which are in turn more effective than symbolic HWLs. We hypothesised that the neural response in our a priori ROIs would differentiate between our three types of HWL (graphic >suffering>symbolic), and that participants who rated pictorial HWL stimuli as more emotionally arousing would exhibit heightened activity in these areas. In order to examine these questions, 50 current adult smokers selfreported emotional arousal elicited by HWLs of each pictorial subtype and subsequently observed the same stimuli while their brain activity was measured using functional MRI (fMRI).
METHOD

\section{Participants}

Fifty adult smokers between the ages of 18 and 50 (24 females, mean age $=27.57$ ) took part in this study. Participants were recruited from the general public, via fliers posted in public locations around the University of South Carolina and local newspapers. All participants were neurologically healthy smokers with normal or corrected to normal vision. Following initial phone and online screening to confirm qualification for participation, all participants reported to the McCausland Center for Brain Imaging and provided informed consent prior to MRI scanning. Following completion of the study protocol, participants were paid US $\$ 100$ for transportation costs related to participation in the study. The experiment was performed according to the guidelines of the Declaration of Helsinki.

\section{Pictorial HWL stimuli}

A total of 57 pictorial HWLs were used, with images drawn primarily from, based on, or considered for actual HWLs implemented in different countries (online supplementary figure S1), including prior HWL research that has relied on self-reported responses to HWLs to determine the efficacy of different content. ${ }^{6}{ }^{29}$ Nineteen pictorial HWLs were developed for each of the three pictorial styles that were matched on textual and topical content: (1) graphic health effect-vivid depiction of physical effects of smoking on the body; (2) human suffering-depiction of personal experience which shows the face and could include the physical, social or emotional impact of smoking-related harm and (3) symbolic-representation of health risks using abstract imagery or symbols. Prior HWL research indicates that adult smokers and adolescents discriminate between these three general categories of HWL imagery. ${ }^{9-13} 30$ The textual content to accompany pictorial elements involved short, factual statements based on HWLs that countries have implemented or that have been used in prior research. ${ }^{9}$ HWL topics addressed 13 different health issues (ie, addiction, death, emphysema, gangrene, heart disease, lung cancer, mouth cancer, pregnancy, breast cancer, secondhand smoke, strokes, throat cancer and blindness), with some topics (emphysema, death, heart disease, lung cancer, mouth cancer, stroke) having two sets of three HWLs on the same health topic but with one of each different pictorial style (graphic, suffering and symbolic). Textual elements were matched across all three HWL subtypes. Importantly, the mean luminance values for pictorial HWLs did not differ between subtypes (all $\mathrm{p}>0.18$ ), nor did the overall colour (as measured by red, green or blue colour values; all $\mathrm{p}>0.11)$.

\section{Study procedures}

\section{Demographic data}

All participants were asked standard questions regarding their age, gender, income, ethnicity, and current and past use of cigarettes (online supplementary table S1). 
Self-reported responses to HWLs

Prior to attending the laboratory session, each participant completed a short survey and rated all 57 HWLs, which were presented online and in random order. The primary reason for collecting the self-report ratings before the functional magnetic resonance imaging (fMRI) experiment was to minimise respondent burden, as the fMRI protocol lasted an hour. We gauged this as a greater concern than familiarisation (which could attenuate subsequent blood oxygen level dependent (BOLD) response), especially as smokers are usually exposed to HWLs many times every day. Negative emotional arousal was assessed by asking participants to rate the HWL on how much it made them afraid ("How much does this warning make you feel afraid?"). As in prior research, ${ }^{9}{ }^{12}$ participants were also queried concerning ad effectiveness ("How effective is this warning?"). For both questions, participants responded with a rating of $1-9$, with verbal anchors at either end of the rating scale (ie, $1=$ not at all, $9=$ extremely).

\section{Smoking status screening}

To confirm smoking status, carbon monoxide (CO) levels were measured in all participants immediately prior to scanning using a piCO+ Smokerlyzer (Bedfont Scientific, Harrietsham, England). All participants also provided saliva samples immediately prior to scanning to assess cotinine (nicotine metabolite) using liquid chromatography with tandem mass spectrometry (LC-MS/ MS). These assays confirmed self-reported smoking status for all participants. Participants also reported the time since last cigarette, the number of days they smoked in the past 30 days and the average number of cigarettes they smoked per day during that time (online supplementary table S1).

\section{Neural response to HWLs}

During 50 min of MRI, each participant completed a single, high-resolution structural scan, as well as four functional MRI task runs. Each functional run was $10 \mathrm{~min}$ and $24 \mathrm{~s}$ in duration. HWLs were presented using a block design format. Each block of stimuli was $15 \mathrm{~s}$ in duration and consisted of the serial presentation of five images from the relevant condition (or fixation cross for Rest), separated by $1 \mathrm{~s}$ of fixation. A total of 40 blocks (10 graphic images, 10 suffering images, 10 symbolic images and 10 Rest) were presented during each of four functional runs, for a total of 150 HWLs per functional run (50 in each category). The 150 images within a given functional run were randomly chosen from a pool of 600 images created at the beginning of the scanning session. This pool of 600 images consisted of 10 of each individual HWL $(10 \times 19 \times 3=570)$, with the remaining 30 being randomly chosen (10 pseudorandom choices from each category-the constraint being that they all had to be different, ie, no repeats within this subset; figure 1). The order of presentation of the blocks within a given functional run was chosen from
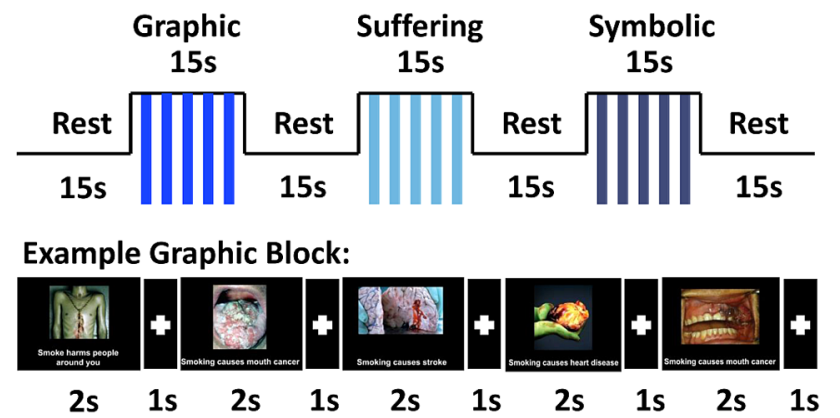

Example Suffering Block:

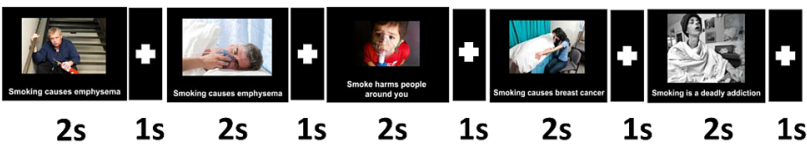

Example Symbolic Block:

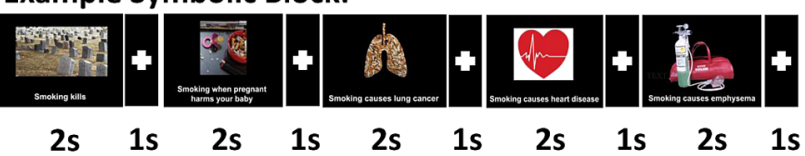

Figure 1 Graphical representation of the construction of each functional run. All stimuli types (graphic, suffering and symbolic) were presented in block format. Each block consisted of the presentation of five pseudorandomly selected stimuli of the appropriate type presented for $2 \mathrm{~s}$ each, and separated by $1 \mathrm{~s}$ of fixation. Block order was pseudorandomized for each functional run.

one of eight pseudorandomly generated trial orders. These orders were constrained such that (1) each condition was equally likely to follow any other condition within a certain functional run and (2) blocks of the same trial type never occurred more than three times in a row. Each of the four functional runs was identical in duration and content with the exception of the random assignment of images from each condition to its corresponding block. Importantly, the total time (and thus total number of brain volumes recorded) spent showing blocks of each picture type was identical to the total time spent showing Rest blocks.

In order to ensure that participants paid attention to the visual stimuli, we employed a one-back picture recognition task. Participants were instructed to press a button when the same picture appeared twice in a row. Each functional run contained either five or six repeated pictures which required the participant to press a button. Placement of repeats was randomised prior to each run using Presentation's built in randomisation subroutines.

\section{fMRI methods}

Image acquisition

All MRI data were collected on a 3T Siemens Trio system with a 12-element head coil. The fMRI $\left(\mathrm{T}_{2} *\right.$ echo planar imaging) imaging sequence included the following parameters: 320 full brain volumes collected in each of the four $10 \mathrm{~min}, 24 \mathrm{~s}$ runs; $75^{\circ}$ flip angle; time 
repetition $(\mathrm{TR})=1.95 \mathrm{~s}$; time echo $(\mathrm{TE})=30 \mathrm{~ms}$; in-plane resolution $3.30 \times 3.30 \mathrm{~mm}$; slice thickness $=3 \mathrm{~mm}$ (no gap); 36 axial slices collected in planes aligned parallel to the anterior-posterior commissure line. To improve coregistration of images, all participants were scanned with a high-resolution $\mathrm{T}_{1}$ MRI, which yielded a $1 \mathrm{~mm}$ isotropic image. This sequence had the following parameters: field of view $(\mathrm{FOV})=256 \times 256 \mathrm{~mm}, 192$ saggital slices, $9^{\circ}$ flip angle, $\mathrm{TR}=2250 \mathrm{~ms}$ and $\mathrm{TE}=4.15 \mathrm{~ms}$.

\section{Data preprocessing and modelling}

All fMRI data were preprocessed and analysed using SPM8 (Wellcome Department of Cognitive Neurology, London). Standard preprocessing procedures included image realignment (4th Degree B-Spline Interpolation), coregistration (mean EPI aligned with T1 then parameters applied to all EPIs), normalisation and spatial smoothing (Gaussian Kernel full width at the half maximum (FWHM) $8 \mathrm{~mm}$ ). The onsets and durations of each of the conditions of interest were modelled according to the block design described in the protocol. For our primary analysis, functional data across the four runs was modelled as a boxcar canonically convolved hemodynamic response function (duration $10 \mathrm{~s}$ ). For results regarding between-run differences (ie, neural adaptation), condition-specific activation within each functional run was modelled as a separate set of events. For all group analyses reported below, we first generated a series contrast images for each individual participant (first-level models) and then entered these into random-effects models and/or regression models (using SPM's built in general linear model) in order to allow for meaningful population-level inference. First eigenvariates were extracted from second-level models (for each ROI/condition/run) using the VOI toolbox in SPM $8 .{ }^{31}$ For the multiple regression analysis between self-reported ratings and neural responses reported below, means for neural responses were calculated at the HWL level (mean values were calculated for each participant for the neural response in each ROI and for each HWL subtype). The resulting parameter estimates were used as the primary dependent variables in the statistical models reported below (ie, analysis of variance (ANOVA) and regression analyses).

\section{RESULTS}

\section{Behavioral performance}

Population variables

Participants in the current study were equally spilt with respect to gender (52\% male and $48 \%$ female) and predominantly white $(74 \%, 24 \%$ African American and 2\% other). The majority of participants $(55 \%)$ had at least some post-high school education and was low income. At the time of scanning, the group's CO levels were $18.74 \mathrm{ppm}$ and cotinine was measured at $207.48 \mathrm{ng} / \mathrm{mm}$ confirming that all participants were active smokers. Furthermore, the average participant smoked 18.74 cigarettes per day, and reported having smoked on 28.32 out of the previous 30 days.

\section{Self-reported ratings of HWLS}

Differences in self-reported emotional arousal across the three stimulus types (graphic, suffering and symbolic) was assessed using one-way within subjects ANOVA, F $(1.44,70.53)=121.01, \mathrm{p}<0.001$. A one-way within subjects ANOVA using perceived effectiveness as a dependent variable and stimulus-type (graphic, suffering and symbolic) as the independent variable was also significant, $\mathrm{F}$ $(1.54,75.27)=133.27, p<0.001$. For both ANOVAS, post hoc pair-wise comparisons revealed significant differences between ratings of graphic and suffering stimuli, as well as between ratings of suffering and symbolic stimuli (all $\mathrm{p}<0.01$ ).

Responses to the emotional arousal and perceived effectiveness questions were highly correlated for the graphic $(\mathrm{r}(49)=0.87)$, suffering $(\mathrm{r}(49)=0.90)$ and symbolic $(r(49)=0.90)$ stimuli. Because ratings of emotionality were the most relevant for interpretation of our results, we focus on those scores in our analysis section. When the same analyses were conducted using perceived effectiveness, we obtained a similar pattern of results (ie, graphic $>$ suffering $>$ symbolic; figure 2 ).

\section{fMRI one-back task}

One-back task performance data was collected from a total of 176 of 200 possible fMRI scanning runs (50 participants, with 4 runs per person). Data from 24 of the runs were lost due to experimenter error. We did not exclude the imaging data from these participants as we did monitor the participants' error rates online and ensure they were paying attention (they were just not recorded). A one-way ANOVA using error rate as the dependent variable and run as the factor was not significant, $\mathrm{F}(3,162)=1.003, \mathrm{p}=0.393$. Moreover, post hoc comparison failed to reveal any significant differences between error rates in any two runs (all $\mathrm{p}>0.33$ ).

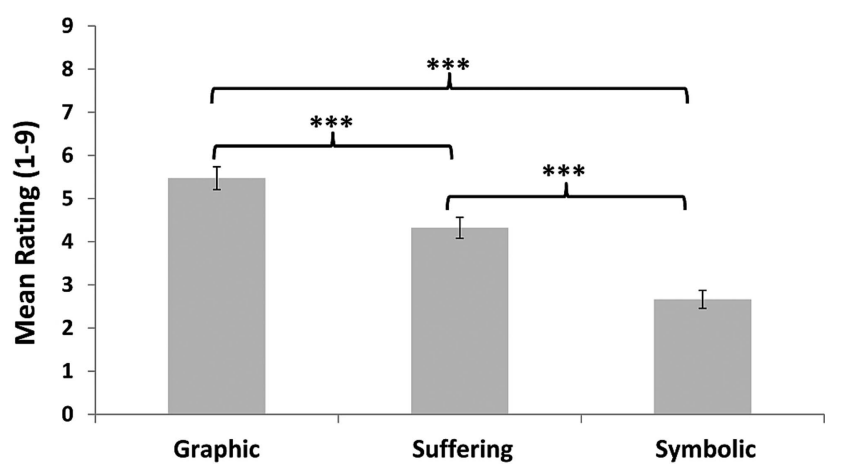

Figure 2 Behavioral effectiveness ratings of health warning labels (HWLs). All participants rated all HWLs prior to functional MRI (fMRI) scanning by responding to the question: "How much does this warning make you feel afraid?"

${ }^{* \star *}$ Significant $p<0.001$ (within subjects one-tailed $t$ test); error bars represent SEM. 
Table 1 Brain regions responding to the presentation of pictorial HWLs in current adult smokers

\begin{tabular}{|c|c|c|c|c|c|}
\hline \multirow[b]{2}{*}{ Region } & \multirow[b]{2}{*}{ L/R } & \multicolumn{3}{|c|}{$\begin{array}{l}\text { Local maxima } \\
\text { peak } \\
\text { Coordinates (MNI) }\end{array}$} & \multirow[b]{2}{*}{ T value } \\
\hline & & $\bar{x}$ & $y$ & $\mathbf{z}$ & \\
\hline \multicolumn{6}{|l|}{ All-Rest } \\
\hline Lingual gyrus & $\mathbf{R}$ & 24 & -90 & -6 & 21.62 \\
\hline Fusiform gyrus & $\mathrm{R}$ & 42 & -80 & -10 & 19.48 \\
\hline Calcarine & $\mathrm{R}$ & 12 & -94 & 0 & 19.02 \\
\hline Hippocampus & $\mathrm{R}$ & 20 & -30 & 0 & 15.8 \\
\hline Hippocampus & $\mathrm{L}$ & -22 & -30 & -2 & 13.73 \\
\hline IFG pars triangularis & $\bar{L}$ & -52 & 24 & 30 & 9.87 \\
\hline Precentral gyrus & $\mathrm{L}$ & -46 & -4 & 52 & 9.71 \\
\hline Precentral gyrus & L & -42 & 8 & 32 & 9.26 \\
\hline SMA & $\mathrm{L}$ & -6 & 8 & 56 & 8.99 \\
\hline SMA & $\mathrm{R}$ & 6 & 10 & 52 & 8.53 \\
\hline IFG pars triangularis & $\mathrm{R}$ & 48 & 24 & 26 & 8.67 \\
\hline IFG pars opercularis & $\mathrm{R}$ & 54 & 22 & 32 & 8.66 \\
\hline Middle frontal gyrus & $\mathrm{R}$ & 50 & 36 & 24 & 8.64 \\
\hline Insula & $\mathbf{L}$ & -30 & 28 & 2 & 8.39 \\
\hline IFG pars orbitalis & $\mathrm{L}$ & -34 & 30 & -8 & 8.17 \\
\hline IFG pars orbitalis & $\mathrm{L}$ & -40 & 26 & -12 & 7.81 \\
\hline Amygdala & $\mathbf{R}$ & 20 & -6 & -14 & 7.33 \\
\hline Amygdala & $\mathbf{L}$ & -22 & -4 & -14 & 6.47 \\
\hline IFG pars orbitalis & $\mathrm{R}$ & 28 & 30 & -10 & 6.12 \\
\hline Insula & $\mathbf{R}$ & 32 & 30 & 2 & 5.57 \\
\hline Fusiform gyrus & $\mathrm{L}$ & -32 & -32 & -16 & 6.02 \\
\hline Parahippocampal gyrus & $\mathrm{L}$ & -14 & -28 & -16 & 5.13 \\
\hline \multicolumn{6}{|l|}{ Graphic-Rest } \\
\hline Lingual gyrus & $\mathbf{R}$ & 24 & -90 & -6 & 19.86 \\
\hline Declive & $\mathrm{L}$ & -38 & -70 & -10 & 19.05 \\
\hline Fusiform gyrus & $\mathrm{R}$ & 42 & -80 & -10 & 18.41 \\
\hline Hippocampus & $\mathrm{L}$ & -22 & -30 & -2 & 11.35 \\
\hline Hippocampus & $\mathrm{R}$ & 22 & -30 & 0 & 13.19 \\
\hline Precentral gyrus & $\mathrm{L}$ & -46 & -4 & 48 & 9.42 \\
\hline Precentral gyrus & $\bar{L}$ & -50 & 6 & 38 & 8.68 \\
\hline Precentral gyrus & $\mathrm{L}$ & -42 & 6 & 32 & 8.47 \\
\hline SMA & $\mathrm{L}$ & -6 & 6 & 58 & 8.54 \\
\hline SMA & $\mathrm{R}$ & 6 & 10 & 52 & 7.87 \\
\hline Precentral gyrus & $\mathrm{R}$ & 46 & 8 & 34 & 8.36 \\
\hline Middle frontal gyrus & $\mathrm{R}$ & 50 & 36 & 24 & 8.31 \\
\hline IFG pars opercularis & $\mathrm{R}$ & 54 & 22 & 30 & 7.91 \\
\hline Insula & $\mathbf{L}$ & -30 & 30 & -4 & 7.46 \\
\hline Parahippocampal gyrus & $\mathrm{R}$ & 36 & -6 & -26 & 6.54 \\
\hline Amygdala & $\mathbf{L}$ & -22 & -2 & -16 & 6.38 \\
\hline Amygdala & $\bar{R}$ & 22 & -4 & -14 & 6.1 \\
\hline Parahippocampal gyrus & $\mathrm{L}$ & -30 & -34 & -16 & 5.94 \\
\hline IFG pars orbitalis & $\mathrm{R}$ & 28 & 30 & -10 & 5.69 \\
\hline Middle temporal gyrus & $\mathrm{L}$ & -54 & -46 & 8 & 5.42 \\
\hline \multicolumn{6}{|l|}{ Suffering-Rest } \\
\hline Fusiform gyrus & $\mathrm{R}$ & 42 & -80 & -10 & 19.19 \\
\hline Lingual gyrus & $\mathbf{R}$ & 24 & -90 & -6 & 19.1 \\
\hline Occipital lobe (middle) & $\mathbf{L}$ & -26 & -96 & 8 & 18.46 \\
\hline Hippocampus & $\mathrm{R}$ & 24 & -28 & -2 & 15.59 \\
\hline Hippocampus & $\mathrm{L}$ & -22 & -28 & -4 & 14.41 \\
\hline Amygdala & $\mathbf{R}$ & 20 & -6 & -14 & 9.36 \\
\hline IFG pars triangularis & $\mathrm{R}$ & 52 & 30 & 26 & 9.05 \\
\hline IFG pars opercularis & $\mathrm{R}$ & 46 & 14 & 32 & 8.54 \\
\hline IFG pars opercularis & $\mathrm{R}$ & 52 & 20 & 34 & 7.88 \\
\hline Insula & $\mathbf{L}$ & -30 & 28 & 0 & 8.65 \\
\hline Inferior frontal gyrus & $\mathrm{L}$ & -36 & 20 & -18 & 5.25 \\
\hline Precentral gyrus & $\mathrm{L}$ & -46 & -4 & 48 & 8.48 \\
\hline Precentral gyrus & $\mathrm{L}$ & -40 & 8 & 32 & 8.42 \\
\hline IFG pars yriangularis & $\mathrm{L}$ & -44 & 18 & 26 & 7.72 \\
\hline SMA & $\mathrm{R}$ & 6 & 10 & 52 & 8.14 \\
\hline
\end{tabular}

Table 1 Continued

\begin{tabular}{|c|c|c|c|c|c|}
\hline \multirow[b]{2}{*}{ Region } & \multirow[b]{2}{*}{ L/R } & \multicolumn{3}{|c|}{$\begin{array}{l}\text { Local maxima } \\
\text { peak } \\
\text { Coordinates (MNI) }\end{array}$} & \multirow[b]{2}{*}{$T$ value } \\
\hline & & $\bar{x}$ & $\mathbf{y}$ & $\mathbf{z}$ & \\
\hline Amygdala & $\mathbf{L}$ & -20 & -6 & -14 & 7.71 \\
\hline Superior temporal gyrus & $\mathrm{L}$ & -52 & -52 & 10 & 7.4 \\
\hline Insula & $\mathbf{R}$ & 30 & 32 & -8 & 6.31 \\
\hline Inferior parietal lobule & $\mathrm{L}$ & -48 & -26 & 52 & 5.56 \\
\hline Superior temporal gyrus & $\mathrm{R}$ & 48 & -40 & 10 & 5.4 \\
\hline \multicolumn{6}{|l|}{ Symbolic-Rest } \\
\hline Lingual gyrus & $\mathbf{R}$ & 24 & -90 & -6 & 19.56 \\
\hline Cuneus & $\mathbf{L}$ & -18 & -100 & 6 & 18.61 \\
\hline Lingual gyrus & $\mathbf{R}$ & 12 & -94 & 0 & 17.98 \\
\hline Hippocampus & $\mathrm{R}$ & 22 & -28 & -2 & 14.14 \\
\hline Hippocampus & $\mathrm{L}$ & -22 & -30 & -2 & 11.36 \\
\hline IFG pars triangularis & $\mathrm{L}$ & -50 & 22 & 30 & 8.92 \\
\hline IFG pars opercularis & $\mathrm{L}$ & -42 & 10 & 30 & 8.57 \\
\hline Precentral gyrus & $\mathrm{L}$ & -46 & -4 & 48 & 8.5 \\
\hline SMA & $\mathrm{L}$ & -4 & 8 & 56 & 8.77 \\
\hline SMA & $\mathrm{R}$ & 6 & 12 & 52 & 8.72 \\
\hline IFG pars opercularis & $\mathrm{R}$ & 54 & 22 & 32 & 7.68 \\
\hline Middle frontal gyrus & $\mathrm{R}$ & 50 & 36 & 24 & 7.59 \\
\hline Precentral gyrus & $\mathrm{R}$ & 46 & 12 & 32 & 6.76 \\
\hline Insula & $\mathbf{L}$ & -30 & 28 & 0 & 7.28 \\
\hline IFG pars orbitalis & $\mathrm{L}$ & -36 & 28 & -10 & 7.2 \\
\hline Inferior parietal lobule & $\mathrm{L}$ & -46 & -38 & 54 & 6.19 \\
\hline Inferior parietal lobule & $\mathrm{L}$ & -48 & -28 & 52 & 5.32 \\
\hline Insula & $\mathbf{R}$ & 32 & 30 & 2 & 5.2 \\
\hline
\end{tabular}

T value: local maxima thresholded at $p<0.05$ FWE corrected, extent threshold $\mathrm{k}=10$.a priori ROls indicated in bold.

FWE, family-wise error; IFG, inferior frontal gyrus; L, left hemisphere; MNI, Montreal Neurological Institute; R, right hemisphere; ROI, regions of interest; SMA, supplementary motor area.

\section{fMRI response}

\section{Primary fMRI outcomes}

\section{Main effects of HWL type}

In order to isolate cortical networks activated by the presentation of each type of pictorial HWL, we computed a series of contrasts designed to test for the main effects of each of the three stimulus types (graphic, suffering and symbolic). Specifically, we computed the following contrasts: graphic-Rest, suffering-Rest and symbolic-Rest (thresholded at $\mathrm{p}<0.05$ and corrected for family-wise error). Observation of pictorial HWL stimuli elicited a significant neural response in a broad network of brain areas including our a priori ROIs (the amygdala, insula and visual association cortex) as well as a number of other brain areas including the frontal gyrus (inferior, middle, medial and superior aspects), temporal gyrus (middle and superior), parietal lobe (inferior), supplementary motor area, parahippocampal gyrus and thalamus. The results of this analysis are listed in table 1 and displayed graphically in figure 3.

\section{Comparison of HWL-elicited activation in a priori ROls}

We performed additional analyses in order to identify brain areas that responded maximally to graphic HWLs, less to suffering HWLs and least to symbolic HWLs. 

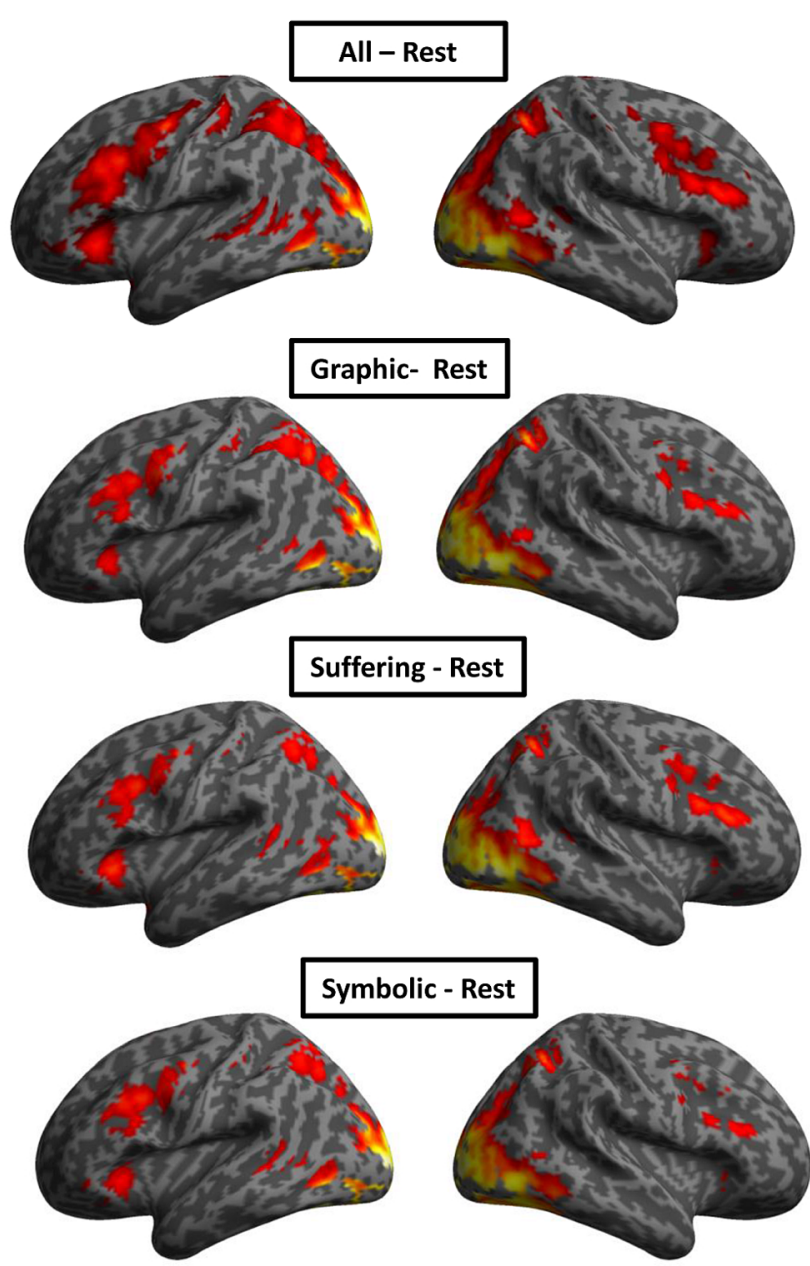

Figure 3 Main effects of health warning labels on BOLD signal (graphic, suffering, symbolic) on BOLD signal. All results are thresholded at $\mathrm{p}<0.05$ and corrected for family-wise error. Results are overlaid on a standard inflated brain (cortex_20484.surf.gii) for illustration purposes.

Accordingly, we performed ROI analyses on our a priori ROIs including the amygdala, insula and visual association cortex. ROIs within these areas were created based on peak activations observed in the contrast comparing the brain's response to all conditions to rest ( (graphic +suffering+symbolic)-Rest). ${ }^{31}$ All ROIs were centred at the site of peak activation within a given ROI and were spherical in nature $(\mathrm{r}=4 \mathrm{~mm})$. A series of one-way withinsubjects ANOVAs were used to evaluate neural responses patterns (for graphic, suffering and symbolic stimuli) within our ROIs. These ANOVAs were significant in the left amygdala, $\mathrm{F}(2,98)=14.59, \mathrm{p}<0.001$; right amygdala, $\mathrm{F}(2,98)=21.60, \mathrm{p}<0.001$; left insula, $\mathrm{F}(2,98)=4.42, \mathrm{p}<0.05$ and visual association cortex, $\mathrm{F}(2,98)=22.69, \mathrm{p}<0.001$. As with the behavioural data, we conducted post hoc pairwise comparisons (all significant results were $\mathrm{p}<0.05$, Bonferroni corrected). In the left amygdala we observed a significant difference between responses in the graphic and symbolic conditions, as well as in the suffering and symbolic conditions. In the right amygdala all pair-wise comparisons were significant. In the left amygdala and the visual association cortex, responses to graphic and symbolic stimuli were significantly different, as were responses to graphic and suffering stimuli. The results of these analyses are shown graphically in figure 4A. We also conducted whole-brain analyses for the following direct comparisons between conditions: graphic $>$ symbolic:symbolic $>$ graphic (online supplementary table S2), suffering $>$ symbolic:symbolic $<$ suffering, (online supplementary table S3) and suffering $>$ graphic: graphic $>$ suffering (online supplementary table S4).

\section{Secondary fMRI outcomes \\ Correlation between self-reported ratings and neural response}

We ran a series of targeted correlations to determine whether there was a relationship between individual ratings of pictorial HWLs of specific subtypes and the BOLD signal elicited by their presentation. For the graphic stimuli, we conducted an SPM multiple regression analysis using individual contrast images for the graphic-Rest condition as the dependent variable and mean self-reported arousal ratings for the graphic HWLs as the independent variable (thresholded at $\mathrm{p}<0.001,5$ voxel extent). Similar regression analyses were conducted to examine the correlation between HWL ratings and BOLD signal in the suffering and symbolic conditions. In all three analyses, activation in the right visual association cortex $\left(\mathrm{XYZ}_{\mathrm{mni}}=\right.$ $-18,-92,20 ; \mathrm{XYZ}_{\mathrm{mni}}=-20,-88,12$ and $\mathrm{XYZ}_{\mathrm{mni}}=-14,-92$, 12 , respectively) was positively correlated with mean ratings of the pictorial HWLs (all $\mathrm{r}$ (49) $>0.48$; figure 5). For graphic and suffering HWLs additional positive correlations were found at sites in the right precentral gyrus $\left(\mathrm{XYZ}_{\mathrm{mni}}=44,4,40\right), \mathrm{r}(49)=0.45$ and $\mathrm{r}(49)=0.42$ respectively. We also found a significant positive correlation between ratings for symbolic HWLs, and BOLD signal elicited by their presentation, in the left inferior frontal gyrus $\left(\mathrm{IFG} ; \mathrm{XYZ}_{\mathrm{mni}}=-52,16,30\right)$, r (49)=0.37).

\section{Exploratory analysis of BOLD signal adaptation}

In addition to examining the main effects of stimulus type, we also conducted a series of 3 (stimulus) $\times 4$ (session) repeated measures ANOVAs (one for each ROI) in order to explore possible BOLD signal adaptation to our three stimuli types across the four fMRI runs. The main effect of run was significant for the left insula, $\mathrm{F}(3,138)=11.40, \quad \mathrm{p}<0.001$, right insula $\mathrm{F}(3,138)=3.19$, $\mathrm{p}<0.05$ and visual association cortex, $\mathrm{F}(3,138)=15.43$, $\mathrm{p}<0.001$, and nearly significant in the left amygdala, $F(3,138)=2.66, p=0.07$. There was a significant interaction between stimulus and run in the left amygdala, $\mathrm{F}$ $(6,276)=2.28, \mathrm{p}<0.05$ and right amygdala, $\mathrm{F}(6,276)=2.15$, $\mathrm{p}<0.05$. These results are shown split by run (in order to visualise adaptation) in figure $4 \mathrm{~B}$.

\section{DISCUSSION}

\section{Self-reported ratings of pictorial HWLs}

Results from the current study were generally consistent with prior research using self-reported responses to 
Figure 4 (A) Results from ROI analyses. (B) Adaptation of BOLD signal in ROls across four functional scanning runs. L_AMG, left amygdala $\left(X Y Z_{m n i}=-26,-2\right.$, -17); R_AMG, right amygdala $\left(X Y Z_{m n i}=23,7,-17\right)$; L_INS, left insula $\left(X Y Z_{m n i}=-30,30,4\right)$;

R_INS, right insula $\left(X Y Z_{m n i}=28\right.$, 32, -8); L_OCC, left occipital cortex $\left(X Y Z_{m n i}=-26,-94,4\right)$; OCC, occipital cortex $\left(X Y Z_{m n i}=\right.$ $-26,-94,4 ; X Y Z_{m n i}=24,-90$, $-6)$; * significant $p<0.05$ (within subjects one-tailed $t$ test);

${ }^{* *}$ significant $p<0.05$, ${ }^{* * *}$ significant $p<0.001$ (within subjects one-tailed $t$ test); error bars represent SEM. ROI, regions of interest.
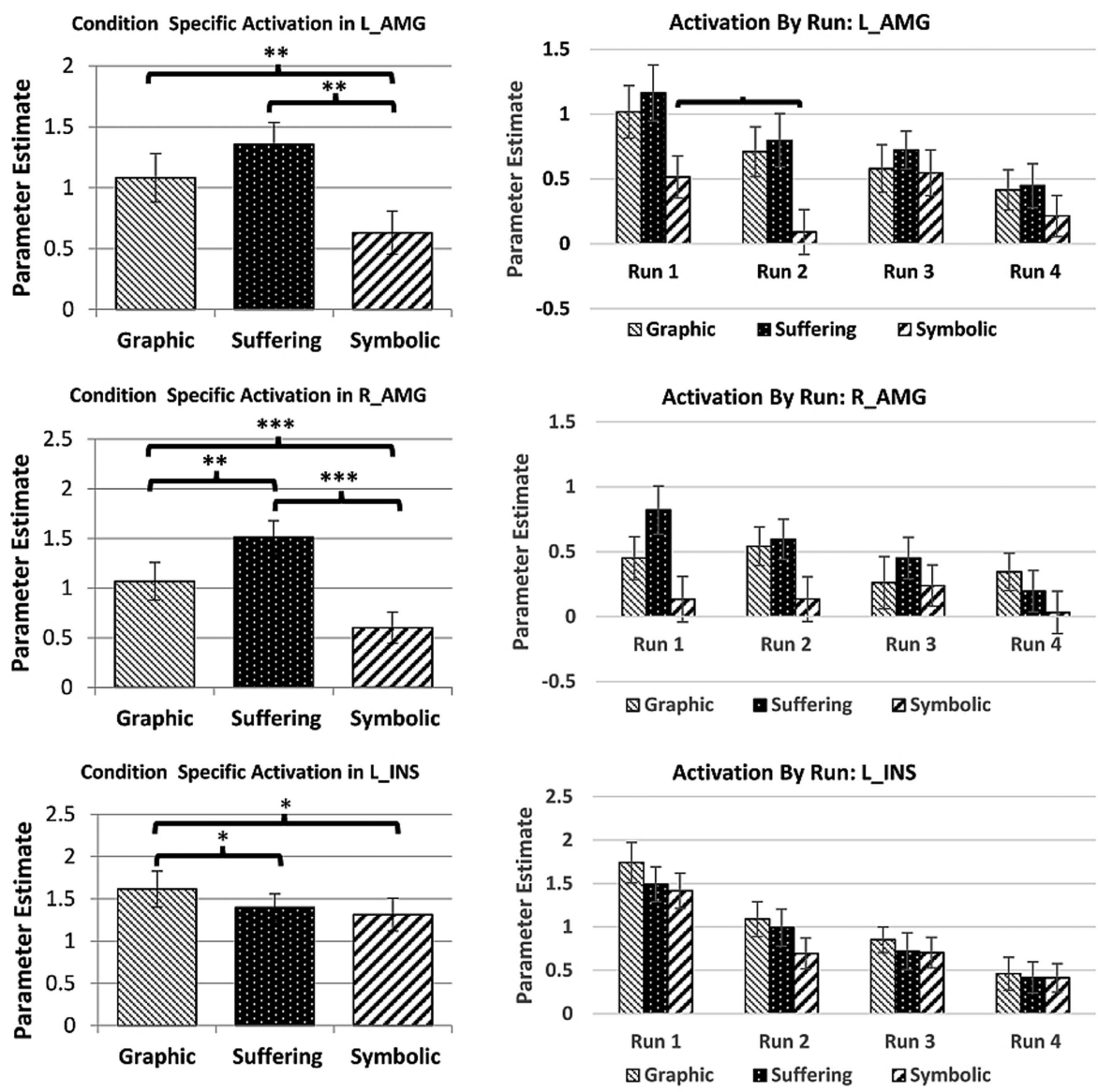

Condition Specific Activation in R_INS
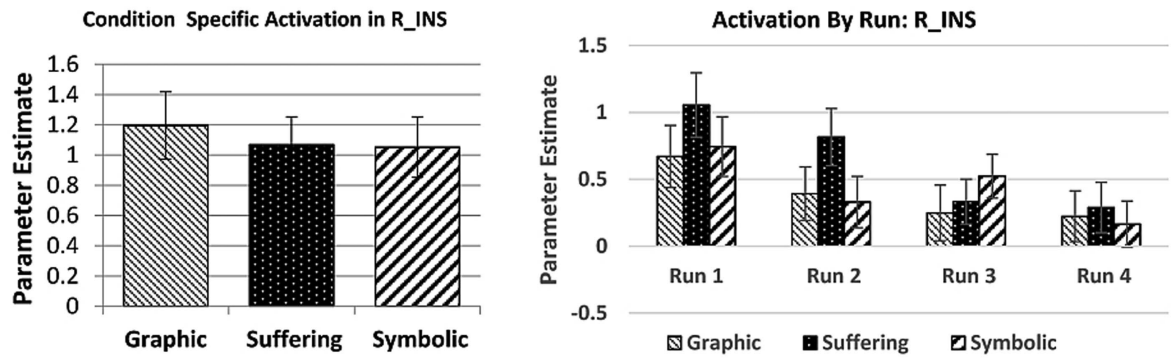

Condition Specific Activation in VAC

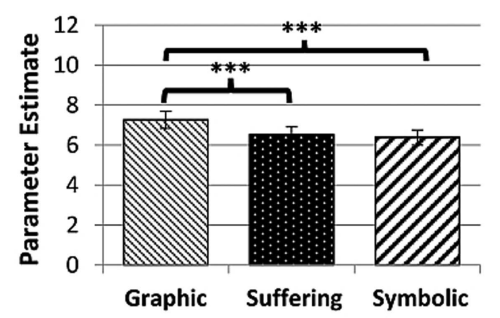

HWL stimuli. This research consistently indicates that smokers report stronger responses to HWLs with graphic imagery than to symbolic imagery. ${ }^{9-13} 32$ Results suggesting the greater impact of imagery of suffering than graphic imagery are not necessarily inconsistent with this research. Indeed, a number of the suffering images included graphic elements, and HWLs that combine the two may be most effective. ${ }^{9}$ Nevertheless, as for selfreport research, future fMRI research is needed to determine whether neural responses predict meaningful behavioural change (ie, quitting smoking) or perceptual change (eg, better understanding of risks, particularly among youth). In general, however, this study suggests that fMRI and self-report produce similar results. One possible concern with the present results is that we did not confirm whether our specific sample of participants considered each pictorial HWL to belong to one category or another. Future research may consider asking 
Correlation Between Self-Reported Ratings of Induced Fear and Activation In Visual Association Cortex

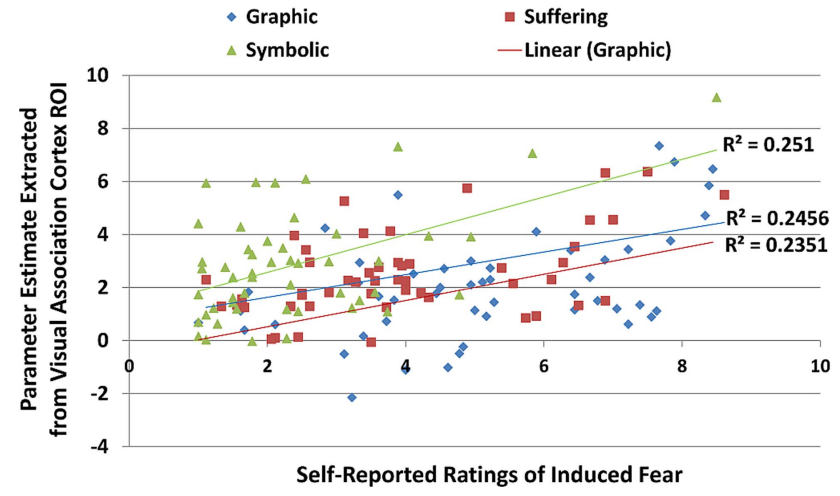

Figure 5 Correlation between BOLD signal in the visual association cortex (BA 18) and participant self-reported ratings of different subtypes of HWL. The site of maximal correlation between the parameter estimates for the contrast (graphic-Rest) and self-reported ratings of graphic $\mathrm{HWL}$ stimuli was located at $\left(X Y Z_{m n i}=-19,-92,20\right)$. The site of maximal correlation between the parameter estimates for the contrast (suffering-Rest) and self-reported ratings of suffering $\mathrm{HWL}$ stimuli was located at $\left(X Y Z_{m n i}=-20,-88,12\right)$. The site of maximal correlation between parameter estimates for the contrast (symbolic-Rest) and self-reported ratings of symbolic $\mathrm{HWL}$ stimuli was located at $\left(X Y Z_{\mathrm{mni}}=-14,-92,12\right)$. HWL, health warning label; ROI, regions of interest.

participants to sort pictorial HWLs into categories to address this concern.

\section{Main effects of HWL type}

The primary goal of the current experiment was to assess neural responses to the presentation of different types of pictorial HWLs that governments have considered for implementation. In general, observation of pictorial HWLs activated large-scale neural networks including the hippocampus, fusiform gyrus, precentral gyrus, supplementary motor area, pars triangularis, pars opercularis, pars orbitalis and fusiform gyrus. Based on prior literature mapping the brain's response to vivid graphic images, we expected all three types of HWLs to elicit activation in the amygdala, the insula and the visual association cortex. Our results are consistent with this literature in that all subtypes of pictorial HWLs used in the current study elicited activation at sites in all three of these areas.

\section{Comparison of HWL-elicited activation in a priori ROls Visual association cortex}

We expected the intensity of BOLD signal in regions associated with visual and emotional processing to mirror self-reported ratings of the stimuli (ie, graphic $>$ suffering $>$ symbolic). Results from our ROI analysis were partially consistent with this prediction. Activity in the right visual association cortex did scale in the same manner as self-reported ratings of the HWL stimuli. The more vivid/graphic nature of certain subtypes of pictorial HWLs may be responsible for the differences we observed in the visual cortex. Images in the graphic condition contained more gory/bloody elements than those in any of the other two conditions; the images in the suffering condition contained a moderate amount of these elements; and images in the symbolic condition contained the least of these elements. We speculate that these negatively valenced elements, which were particularly arousing, may have increased signal in visual areas via afferent projections from the amygdala. It is well established that the amygdala, a key neural pathway for responses to graphic imagery, projects to primary and secondary visual cortices. ${ }^{33}$ It is particularly unlikely that heightened activation in the visual association cortex was caused by differences in low-level features of the images as neither luminance nor colour values for HWL stimuli were significantly different across the three HWL subtypes. Additionally, in at least one previous experiment examining the impact of arousing visual stimuli on visual cortex activity, differences in eye movements did not account for the observed patterns of activation. ${ }^{27}$ Therefore it is unlikely that the effects we report were due to differential eye movements.

\section{Amygdala}

While responses in the visual association area and insula were consistent with self-reported ratings, activation patterns observed in amygdala were not. Unexpectedly, the amygdala was most robustly activated by suffering HWLs, followed by graphic HWLs and finally symbolic HWLs. As noted in the introduction, the amygdala has been shown to be responsive to arousing stimuli, and fearevoking stimuli robustly activate this brain structure. One possibility, then, is that the HWLs depicting personal suffering from smoking-related outcomes are effective at eliciting fear in current adult smokers. However, this is inconsistent with the self-reported data, which indicated that graphic HWLs elicited maximal fear responses. A more parsimonious explanation for this finding is that the relatively higher activation observed for HWLs with suffering imagery was due to the presence of human faces in the stimuli (all 19 suffering HWLs contained human faces). Lesion, single-cell and whole-brain neuroimaging experiments are consistent with the idea that the amygdala is a key component of the face-perception network. ${ }^{17}$ 34-39 The amygdala may even process fearful facial stimuli in the absence of conscious processing. ${ }^{40}{ }^{41}$ Hence, the inclusion of faces may be particularly important to maintaining arousalinducing responses under conditions of repeated exposure, as is typically the case with HWLs. Indeed, recent evidence suggests that sustained responses to repeated presentation of emotional faces may be particularly dependent on the amygdala. ${ }^{42}$ It is also important to note that some of the suffering images (4 of 19) portrayed visible body damage, and thus suffering imagery was not entirely distinct from graphic imagery used in 
the current experiment, and research based on selfreported ratings indicated that this combination produces the strongest ratings. ${ }^{9}$ To better isolate any differential effects of these two image types, as well as the interaction between them, future studies should use imagery that more clearly falls into one category, the other or both. Another possible explanation for the increased relatively higher amygdala activation observed in the suffering condition relates to stimulus salience. Studies have demonstrated a strong link between amygdala activation and this attribute. ${ }^{43} 44$

While these results could have implications for the optimisation of HWLs, further experiments are necessary to evaluate the predictive validity of fMRI. Future research should aim to separate out the effects of emotionality, salience and human faces by integrating additional conditions (such as neutral images with and without faces). On the basis of research demonstrating that the BOLD signal in the amygdala is a predictor of subsequent quitting behaviour ${ }^{45}$ (as is BOLD signal in the medial prefrontal cortex ${ }^{46} 47$ ), future prospective studies should examine the extent to which amygdalar BOLD response to the three types of HWLs discussed in the current paper predict changes in smoking behaviour or, among youth, perceptions of smoking-related risks. Little research has been conducted with youth before they start smoking, and the strongest effects of HWLs may be due to enhancing aversion for smoking as opposed to changing the behaviours of addicted smokers.

\section{Secondary fMRI outcomes}

\section{Correlation between self-reported ratings and neural} response

An important goal of the present study was to crossvalidate self-reported ratings of pictorial HWLs and brain activity recorded during the observation of the same stimuli. This paper is the first to report such results for cigarette HWL stimuli. Regarding correlations between self-reported ratings of HWL stimuli and neural activity in our three a priori ROIs, only the visual cortex was significant (with the amygdala being nearly significant at $\mathrm{p}=0.07$ ). We also report significant correlations between behavioural ratings and two additional areas, the junction of the right precentral and IFG, and the left IFG pars opercularis.

\section{Visual association cortex}

Our correlational data indicate that participants who rated pictorial HWL stimuli (within each category-as opposed to between categories) as more emotionally arousing showed higher activation of the visual association cortex when viewing the stimuli. This finding is consistent with previous reports demonstrating that activity in the visual cortex is particularly robust during the presentation of emotionally arousing visual stimuli, perhaps due to re-entrant enhancement of V2 activity being driven by motivational processes that heighten input from the amygdala. ${ }^{26} 2848$

\section{Insula and amygdala}

Surprisingly, we did not observe a significant correlation between BOLD signal in the insula or amygdala and selfreported ratings of arousal. However, the correlation between BOLD signal in the right amygdala and selfreported responses in graphic $(\mathrm{r}(49)=0.21, \mathrm{p}=0.07$ onetailed) and suffering ( $\mathrm{r}(49)=0.20, \mathrm{p}=0.08$ one-tailed) conditions was nearly significant. This failure to reach statistical significance may be due to a number of factors. One possibility is that the amygdala's response to the emotional stimuli was blunted by the inclusion of text in the HWLs used in the present study. This interpretation is consistent with a comprehensive metaregression analysis of imaging studies on amygdala activation, which found that presence of language in the stimulus was associated with reduced amygdala activation (as well as greater left lateralisation relative to baseline). ${ }^{49}$ While the inclusion of text in graphic warning labels has traditionally been justified in terms of added information content (text adds information otherwise not present), it may also be important to examine possible emotional 'blunting' effects that its inclusion may have. Future brain imaging studies might explore this possibility by simultaneously monitoring brain activity and gaze behaviour. A better understanding of the how people process graphical and textual elements of HWLs, and how attention to one or the other affects neural processing, particularly after repeated HWL exposure that simulates naturalistic exposure conditions, may help inform the design of future HWLs.

\section{Junction of right precentral gyrus and IFG}

We also observed an unexpected correlation between self-report ratings and activity at the junction of the right precentral gyrus and IFG (pars opercularis) for suffering HWLs only. Given the location of the activation in the right hemisphere (as opposed to the left hemisphere which is traditionally associated with such language functions), it is unlikely that heightened responses reflect increased reliance on language. This site is considered to be part of the human mirror neuron system and thought to interact with the amygdala and insula when a link is established between the actions/emotions/intentions of others and our own actions. ${ }^{50}$ One possible explanation for this finding is that suffering stimuli may have been particularly effective at eliciting the types of interpersonal comparisons and or emotions (ie, empathy) that individuals typically make when seeing the negative effects of their own behaviours in others. ${ }^{47}{ }^{51-53}$ Another possible explanation for the significant correlation we observed between right IFG activity and self-reported ratings is that more emotionally arousing stimuli required greater emotion regulation on the part of the observer. This is consistent with studies 
reporting recruitment of the right IFG during tasks that require the inhibition of emotions. ${ }^{54-56}$

\section{IFG, pars opercularis}

Finally, we observed a significant relationship between activity in the left IFG (BA 44) and self-report ratings of the symbolic stimuli. This area has traditionally been associated with language processing and is active during overt (ie, spoken) and covert (ie, silent) speech. ${ }^{57-61}$ It is not surprising that symbolic stimuli would utilise language processes. Stimuli of this subtype were the most abstract and likely evoked covert speech during the interpretation process. The involvement of language areas during HWL processing could be the topic of future experiments that assess verbalisation during presentation of HWLs of all types. While it is reasonable to expect that activation of language areas during HWL processing (an indirect measure of covert verbalisation) may be related to subsequent behavioural change, future studies will need to address this possibility.

\section{Exploratory analysis of BOLD signal adaptation}

To the extent that HWL effectiveness depends on enduring emotional responses, neural adaptation to repeated exposure may be an important issue to consider. Our exploratory, post hoc analysis of region-specific adaptation revealed that, in the majority of our ROIs, BOLD response decreased as a function of repeated exposure to all HWLs. Interestingly, we observed a significant deviation from this pattern in the left and right amygdala. While activation associated with the observation of graphic and suffering images was higher overall, it consistently decreased across the four runs, whereas activation patterns associated with the observation of symbolic images was, overall, less robust and less consistent (figure 4B). Hence, participants may not have adapted (neurally speaking) to repeated presentation of symbolic stimuli in the same way they adapted to images in the suffering and graphic categories. The abstract nature of symbolic stimuli may have required additional exposures in order to more fully process their meaning, and this may account for the observed findings. These data should be interpreted cautiously, however, as repeated exposure to HWLs during three, 10 min scanning runs is unlikely to accurately mimic repeated exposure to HWLs as in real-life, which is temporally spread out, situation specific and associated with cravings and branding imagery that weakens HWL effects. Future research should more directly examine the relationship between the strength of brain activity elicited by specific subtypes of pictorial HWLs after repeated exposures to HWLs, including more naturalistic exposures that allow for adaptation and habituation.

\section{Possible implications for public health policy and} limitations

Understanding how the brain responds to HWLs can inform the optimal development of HWLs. For example, studies on smokers' neural responses to different types of antismoking ads have found that the strength of neural responses elicited by health messaging predict i) subsequent individual-level behavioural change and ii) population-level efficacy of these ads once they are aired in media campaigns. ${ }^{48} 62$ While the current study does not report on behavioural change, future research should. Furthermore, if predictive validity of these methods is established, they could be used to assess the behavioural effects of other types of HWL content. The costeffectiveness of fMRI compared with self-report studies should also be assessed, particularly if they provide consistent results, as we have found here. Data regarding neural adaptation caused by repeated exposure to pictorial HWLs could also be important in terms of informing the creation of HWLs designed for maximum long-lasting impact. Arguably, HWLs will only be effective to the extent that they continue to elicit responses from the consumer. Indeed, the motivation to process messages changes over time, as does the motivation to quit smoking ${ }^{63}$ and HWLs effects may become more potent as these motivations change. Knowing more about the process of adaptation to different types of HWL content, including potential differences in the processes of adaptation across diverse groups, may help with designing HWLs that are most likely to discourage smoking.

\section{General conclusion}

The present study examined adult smokers' self-reported and neural responses to three different types of pictorial HWL stimuli that governments commonly use on cigarette packaging. Pictorial HWLs elicited robust responses in an extensive network of brain sites including those associated with image interpretation (visual association cortex) and emotion (amygdala and insula). Moreover, activation in visual, premotor, inferior frontal and, to a lesser extent, the insular areas, varied in a manner consistent with self-reported ratings of the stimuli. We report a robust relationship between self-reported ratings of arousal and neural responses, which is important considering that self-reported data can be subject to bias. Our exploratory, post hoc analysis of BOLD signal attenuation across scanning runs revealed differences in the patterns of neural adaptation for different types of HWLs that may be relevant to the optimisation of future HWLs. Gaining a better grasp of the relationship between self-reported ratings of HWLs, neural responses elicited by HWLs and the effectiveness of HWLs should be an important goal of future research.

\section{Author affiliations}

${ }^{1}$ Department of Exercise Science, University of South Carolina, Columbia, South Carolina, USA

${ }^{2}$ Department of Health Promotion, Education and Behavior, University of South Carolina, Columbia, South Carolina, USA

${ }^{3}$ Department of Psychology, University of South Carolina, Columbia, South Carolina, USA

${ }^{4}$ Department of Neurosciences, Medical University of South Carolina, Charleston, South Carolina, USA 
${ }^{5}$ Medical University of South Carolina, Hollings Cancer Center, Charleston, South Carolina, USA

${ }^{6}$ University of Waterloo, School of Public Health and Health Systems, Waterloo, Ontario, Canada

Acknowledgements This work was funded by the National Cancer Institute as part of an administrative supplement (P01 CA138389). The authors would like to acknowledge Chris Rorden and Phillip Riddle for their assistance in designing and executing the experiment.

Contributors RDN-N helped design the experiment, collected MRI data, analysed behavioural and MRI data, conducted statistical analyses and drafted the paper. JFT oversaw the experiment, helped design the experiment and helped draft the paper. JF helped design the experiment, recruited participants, collected behavioural and MRI data and revised the draft paper WB helped collect MRI data and revised the draft paper. BEF, DH and MKC helped design the experiment and draft the paper.

Funding This work was supported by National Cancer Institute as part of an administrative supplement (P01 CA138389).

Competing interests None.

Patient consent Obtained.

Ethics approval University of South Carolina IRB.

Provenance and peer review Not commissioned; externally peer reviewed.

Data sharing statement All data collected in this experiment is located on the hard drive of the corresponding author, RDN-N. Data analysis is ongoing and the data may still be used for additional papers. After all planned papers have been submitted, the data may be made available to others, on written request, from RDN-N.

Open Access This is an Open Access article distributed in accordance with the Creative Commons Attribution Non Commercial (CC BY-NC 4.0) license, which permits others to distribute, remix, adapt, build upon this work noncommercially, and license their derivative works on different terms, provided the original work is properly cited and the use is non-commercial. See: http:// creativecommons.org/licenses/by-nc/4.0/

\section{REFERENCES}

1. Adkison SE, O'Connor RJ, Bansal-Travers M, et al. Electronic nicotine delivery systems: international tobacco control four-country survey. Am J Prev Med 2013;44:207-15.

2. WHO. WHO framwork convention. 2009.

3. Olasky SJ, Levy D, Moran A. Second hand smoke and cardiovascular disease in low and middle income countries: a case for action. Glob Heart 2012;7:151-60.e5.

4. Borland R, Wilson N, Fong GT, et al. Impact of graphic and text warnings on cigarette packs: findings from four countries over five years. Tob Control 2009;18:358-64.

5. Fong GT, Hammond D, Hitchman SC. The impact of pictures on the effectiveness of tobacco warnings. Bull World Health Organ 2009;87:640-3.

6. Hammond D, Fong GT, Borland R, et al. Text and graphic warnings on cigarette packages: findings from the international tobacco control four country study. Am J Prev Med 2007;32:202-9.

7. Hammond D, Fong GT, McNeill A, et al. Effectiveness of cigarette warning labels in informing smokers about the risks of smoking: findings from the International Tobacco Control (ITC) Four Country Survey. Tob Control 2006;15(Suppl 3):iii19-25.

8. Kees J, Burton S, Andrews JC, et al. Tests of graphic visuals and cigarette package warning combinations: implications for the framework convention on tobacco control. J Public Policy Mark 2006;25(Fong 2003):212-23.

9. Hammond D, Thrasher J, Reid JL, et al. Perceived effectiveness of pictorial health warnings among Mexican youth and adults: a population-level intervention with potential to reduce tobacco-related inequities. Cancer Causes Control 2012;23(Suppl 1):57-67.

10. Thrasher JF, Allen B, Antrop M, et al. Análisis del impacto en fumadores mexicanos de los avisos gráficos en las cajetillas de cigarros. Salud pública Méx 2006;48:65-74.
11. Thrasher JF, Carpenter MJ, Andrews JO, et al. Cigarette warning label policy alternatives and smoking-related health disparities. Am J Prev Med 2012;43:590-600.

12. Thrasher JF, Arillo-Santillán E, Villalobos V, et al. Can pictoria warning labels on cigarette packages address smoking-related health disparities? Field experiments in Mexico to assess pictorial warning label content. Cancer Causes Control 2012;23(Suppl 1):69-80.

13. Thrasher JF, Villalobos V, Szklo A, et al. Assessing the impact of cigarette package health warning labels: a cross-country comparison in Brazil, Uruguay and Mexico. Salud pública Méx 2010;52(Suppl 2): S206-15.

14. Phelps EA, LeDoux JE. Contributions of the amygdala to emotion processing: from animal models to human behavior. Neuron 2005;48:175-87.

15. Phan KL, Wager T, Taylor SF, et al. Functional neuroanatomy of emotion: a meta-analysis of emotion activation studies in PET and fMRI. Neurolmage 2002;16:331-48.

16. Calder AJ, Lawrence AD, Young AW. Neuropsychology of fear and loathing. Nat Rev Neurosci 2001;2:352-63.

17. Broks $\mathrm{P}$, Young AW, Maratos EJ, et al. Face processing impairments after encephalitis: amygdala damage and recognition of fear. Neuropsychologia 1998;36:59-70.

18. Zald $\mathrm{DH}$. The human amygdala and the emotional evaluation of sensory stimuli. Brain Res Brain Res Rev 2003;41:88-123.

19. Davis M. The role of the amygdala in fear and anxiety. Annu Rev Neurosci 1992;15:353-75.

20. LeDoux J. The emotional brain, fear, and the amygdala. Cell Mol Neurobiol 2003;23:727-38.

21. Maren S, Quirk GJ. Neuronal signalling of fear memory. Nat Rev Neurosci 2004;5:844-52.

22. Stark R, Zimmermann M, Kagerer S, et al. Hemodynamic brain correlates of disgust and fear ratings. Neurolmage 2007;37:663-73.

23. Suzuki A. [Insula and disgust]. Rinsho shinkeigaku 2010;50:1000-2.

24. Schienle A, Schafer A, Hermann A, et al. fMRI responses to pictures of mutilation and contamination. Neurosci Lett. 2006;393:174-8.

25. Murphy FC, Nimmo-Smith I, Lawrence AD. Functional neuroanatomy of emotions: a meta-analysis. Cogn Affect Behav Neurosci 2003;3:207-33.

26. Fredrikson M, Gustav W, Greitz T, et al. Regional cerebral blood flow during experimental phobic fear. Psychophysiology 1993;30:126-30.

27. Lang PJ, Bradley MM, Fitzsimmons JR, et al. Emotional arousal and activation of the visual cortex: an fMRI analysis. 1998.

28. Rauch SL, Bessel A, Fisler RE, et al. A symptom provocation study of posttraumatic stress disorder using positron emission tomography and script-driven imagery. Arch Gen Psychiatry 1996;53:380-7.

29. Canadian Cancer S. Cigarette Package Health Warnings International Status Report. 2012.

30. Hammond D, Reid JL, Driezen $\mathrm{P}$, et al. Pictorial health warnings on cigarette packs in the United States: an experimental evaluation of the proposed FDA warnings. Nicotine Tob Res 2013;15:93-102.

31. Newman-Norlund RD, Ganesh S, van Schie HT, et al. Self-identification and empathy modulate error-related brain activity during the observation of penalty shots between friend and foe. Soc Cogn Affect Neurosci 2009;4:10-22.

32. Cameron LD, Pepper JK, Brewer NT. Responses of young adults to graphic warning labels for cigarette packages. Tob Control 2013. In press.

33. Iwai E YM. Amygdalofugal and amygdalopetal connections with modality-specific visual cortical areas in macaques (Macaca fuscata, M. mulatta, and M. fascicularis). J Comp Neurol 1987;261:362-87.

34. Cannistraro PA, Wright $\mathrm{Cl}$, Wedig MM, et al. Amygdala responses to human faces in obsessive-compulsive disorder. Biol Psychiatry 2004;56:916-20.

35. Rutishauser U, Tudusciuc O, Neumann D, et al. Single-unit responses selective for whole faces in the human amygdala. Curr Biol 2011;21:1654-60.

36. Adolphs R. Neural systems for recognizing emotion. Curr Opin Neurobiol 2002;12:169-77.

37. Adolphs R. Fear, faces, and the human amygdala. Curr Opin Neurobiol 2008;18:166-72.

38. Adolphs R, Gosselin F, Buchanan TW, et al. A mechanism for impaired fear recognition after amygdala damage. Nature 2005;433:68-72.

39. Rapcsak SZ, Galper SR, Comer JF, et al. Fear recognition deficits after focal brain damage: a cautionary note. Neurology 2000;54:575-81.

40. Jiang Y, He S. Report cortical responses to invisible faces: dissociating subsystems for facial-information processing. Perception 2006;16:2023-9. 
41. Pegna AJ, Khateb A, Lazeyras F, et al. Discriminating emotional faces without primary visual cortices involves the right amygdala. Nat Neurosci 2005;8:24-5.

42. Wedig MM, Rauch SL, Albert MS, et al. Differential amygdala habituation to neutral faces in young and elderly adults. Neurosci Lett 2005;385:114-19.

43. Sander D, Grafman J, Zalla T. The human amygdala: an evolved system for relevance detection. Rev Neurosci 2003;14:303-16.

44. Santos A, Mier D, Kirsch P, et al. Evidence for a general face salience signal in human amygdala. Neurolmage 2011;54:3111-16.

45. Jasinska AJ, Chua HF, Ho SS, et al. Amygdala response to smoking-cessation messages mediates the effects of serotonin transporter gene variation on quitting. Neurolmage 2012;60:766-73.

46. Chua HF, Ho SS, Jasinska AJ, et al. Self-related neural response to tailored smoking-cessation messages predicts quitting. Nat NeurosCi 2011;14:426-7.

47. Falk EB, Berkman ET, Whalen D, et al. Neural activity during health messaging predicts reductions in smoking above and beyond self-report. Health Psychol 2011;30:177-85.

48. Lang PJ, Bradley MM, Fitzsimmons JR, et al. Emotional arousal and activation of the visual cortex: an fMRI analysis. Psychophysiology 1998;35:199-210.

49. Costafreda SG, Brammer MJ, David AS, et al. Predictors of amygdala activation during the processing of emotional stimuli: a meta-analysis of 385 PET and fMRI studies. Brain Res Rev 2008;58:57-70.

50. Pfeifer JH, lacoboni M, Mazziotta JC, et al. Mirroring others' emotions relates to empathy and interpersonal competence in children. Neurolmage 2008;39:2076-85.

51. Carr L, lacoboni M, Dubeau M, et al. Neural mechanisms of empathy in humans: a relay from neural systems for imitation to limbic areas. Proc Natl Acad Sci USA 2003;100:5497-502.
52. Leslie KR, Johnson-Frey SH, Grafton ST. Functional imaging of face and hand imitation: towards a motor theory of empathy. Neurolmage 2004;21:601-7.

53. Gallese V, Keysers C, Rizzolatti G. A unifying view of the basis of social cognition. Trends Cogn Sci 2004;8:396-403.

54. Payer DE, Baicy K, Lieberman MD, et al. Overlapping neural substrates between intentional and incidental down-regulation of negative emotions. Emotion 2012;12:229-35.

55. Tabibnia G, Monterosso JR, Baicy K, et al. Different forms of self-control share a neurocognitive substrate. J Neurosci 2011;31:4805-10.

56. Berkman ET, Burklund L, Lieberman MD. Inhibitory spillover: intentional motor inhibition produces incidental limbic inhibition via right inferior frontal cortex. Neurolmage 2009;47:705-12.

57. Numminen J, Curio G. Differential effects of overt, covert and replayed speech on vowel-evoked responses of the human auditory cortex. Neurosci Lett 1999;272:29-32.

58. Paulesu E, Frith CD, Frackowiak RSJ. The neural correlates of the verbal component of working memory. Nature 1993;362:342-5

59. Rueckert L, Appollonio I., Grafman J. Magnetic resonance imaging functional activation of left frontal cortex during covert word production. J Neuroimaging 1994;4:67-70.

60. Price CJ. A review and synthesis of the first 20 years of PET and $\mathrm{fMRI}$ studies of heard speech, spoken language and reading. Neuroimage 2012;62:816-47.

61. Munhall KG. Functional imaging during speech production. Acta Psychol (Amst) 2001;107:95-117.

62. Falk EB, Berkman ET, Lieberman MD. From neural responses to population behavior: neural focus group predicts population-level media effects. Psychol Sci 2012;23:439-45.

63. West R, Sohal T. "Catastrophic" pathways to smoking cessation: findings from national survey. BMJ 2006;332:458-60. 